\title{
SURVEY OBSERVATIONS OF EMISSION LINE STARS AND HERBIG-HARO OBJECTS AT THE KISO OBSERVATORY
}

\author{
K. ISHIDA \\ Institute of Astronomy, University of Tokyo \\ 2-21-1 0sawa, Mitaka, Tokyo 181 Japan
}

ABSTRACT. Summary of the works on emission line stars and Herbig-Haro objects done by visiting astronomers at $K$ iso is presented.

\section{Introduction}

Kiso Observatory was founded in 1974 to operate Schmidt telescope with a corrector of $105 \mathrm{~cm}$ diameter, $150 \mathrm{~cm}$ main mirror, and $330 \mathrm{~cm}$ focal length. Since then, the Kiso Schmidt took 6000 photographs on plates purchased from Eastman Kodak. All the plates taken by the Kiso Schmidt and the image data processing system including several measuring machines are kept at the Observatory site on the mountain. The number of resident staff member is seven, three astronomers and four technicians. The Kiso 0bservatory accepts about 45 scientific programs and about 1000 man-day per year visitings of astronomers from domestic and foreign institutes in current years. The objectives distribute in 1) galaxies and clusters of galaxies (15), 2) nebulae and star clusters (10), 3) stars and stellar objects (9), 4) solar system and position astronomy (8), 5) others (3). One third of the visitings is occupied by those of graduate students guided by the resident astronomers at $\mathrm{Kiso}$. The main program of the $\mathrm{Kis}$ astronomers is isophotometry of a large number of galaxies in current years. Nevertheless, survey observations of emission line stars (ELS) and Herbig-Haro (HH) objects have been actively carried out by the two groups of astronomers with the collaboration of the staff members at Kiso. In the following sections, some of the results of survey observations of ELS and HH objects done by the two groups of astronomers are summarized.

\section{Works done by Xogure's group}

The star forming activity is intimately related to the formation and evolution of galaxies, because the conversion of masses from gas into stellar objects realizes the long lived galaxies existing safely against interaction with other intergalactic objects. In this respect, we can identify three problems that are the mass spectrum at the time of star formation in a particular site, the time dependence of star formation in the Galaxy, and the efficiency of the conversion 
of gas into stars.

ELS are promising candidates of $T$ Tau type stars, which are pre-main sequence stars of intermediate masses, in contrast to that $O B$ stars are young massive stars. By extending survey observations of ELS to as wide area as those done for $O B$ stars (Warren and Hesser 1977), we may probably look at the difference of mass spectra with which stars form from dense cloud and from less-dense cloud.

The adopted method to list up ELS is to take plates by the Kiso Schmidt attached with 4 degree objective prism on Kodak 103aE (hypersensitized) behind Schott RG610. The spectra on the plates are about $1 \mathrm{~mm}$ long between $\lambda \lambda 6100-$ $6800 \mathrm{~A}$, which is long enough to detect $\mathrm{H} \alpha$ emission line (Wiramihardja et al. 1986. Wiramihardja et al. 1989. Kogure et al. 1989).

In the Orion region of 100 square degree area, 1070 ELS with magnitude range of $V=13-17$ were found. The galactic latitude and distance $500 \mathrm{pc}$ of the $0 \mathrm{rion}$ $O B$ association (Warren and Hesser 1977) suggest that the ELS are more than 100 pc below the galactic plane, and the ELS can not be farther away than the 0rion $O B$ association. The distance modulus $m-M=8.5$ and the estimated interstellar extinction $A v=1.0$ indicate that most of the ELS are of $T$ Tau type. If so, the number ratio of ELS to $O B$ stars in different places tells us difference of the mass spectrum with which stars form. The surface number densities of Ori OB1 members and of ELS are both higher in regions of high gas density cloud shown in the CO map (Maddalena et al. 1986) and in the Av map (Tomita, private communication). Remarkable thing is that the ratio of ELS to Ori OB members in surface number is about ten times higher in the less-dense part of dark cloud than in the dense part in the 0rion region. In another word, the stars of intermediate masses are found in a wide area, whereas the massive stars are apparently found in a more restricted region of high gas density. This difference of number ratio of ELS to $O B$ association member in place to place can simply be interpleted as the difference of mass spectrum correlated to the gas density of its parental cloud, if the ages of Ori OB association members and of ELS are nearly the same, and the life times of the both kinds of stars are again nearly the same.

However, there is a hint to indicate that there is a statistical difference in ages of ELS in its parental cloud of different densities. The ELS are classified into 6 grades $(0-5)$ by relative intensities of $\mathrm{H} \alpha$ emission. Fraction of ELS with 3 (medium), 4(strong), and 5 (very strong) $\mathrm{H} \alpha$ is much larger in the region of dense cloud. Further study is needed to draw a firm conclusion on the mass spectrum and time dependence of star formation in the orion region. The catalog of 1070 ELS to list coordinates $\alpha, \delta$, visual magnitude and $\mathrm{H} \alpha$ relative intensity is in preparation and can be obtained from Kogure, Kyoto University.

\section{Works done by Ogura's group}

ELS were searched on $103 \mathrm{aE}$ plates (hypersensitized) taken through RG645 by the Kiso Schmidt attached with the 4 degree objective prism in regions in and around 39 Bok globules of about 11 square degrees (Ogura and Hasegawa 1983, Ogura and Hidayat 1985), in Mon OB1/R1 of about $7 \mathrm{sq} \mathrm{deg} \mathrm{(Ogura} \mathrm{1984),} \mathrm{and} \mathrm{in} \mathrm{dark} \mathrm{cloud}$ Lynds 1228 of about $7 \mathrm{sq}$ deg (Sato et al. 1989). The total number of ELS detected and suspected is 295 , of which 220 are ELS newly discovered.

A new technique was employed to search for $H H$ objects in a wide area from 
homogeneous material. Two exposures are given on a plate with a small displacement behind two different filters one after the other, with a convenient mechanism to exchange a glass filter in front of loaded plate during the telescope is tracking on any sky area. The combinations of emulsion $103 \mathrm{aF}$ (hypersensitized) and filters RG645 and RG610 are utilized. The exposure times were $60 \mathrm{~min}$ and $35 \mathrm{~min}$ in most cases to give equal brightness side by side for ordinary objects, whereas unequal for $H H$ objects with $H \alpha$ emission. Slit spectroscopic observations or CCD direct image observations behind interference filters is needed to segregate HH objects from reflection nebulae out of the survey listings of the Kiso Schmidt. The number of HH object candidates found in M42. NGC 2264, NGC 7822, and NGC 1499 was 18 (0gura 1987) and 10 of which were confirmed to be bona fide HH objects (Ogura and Walsh 1989, Walsh and Ogura 1989).

About $900 \mathrm{HH}$ candidates in M42/L1641, about 60 in Ori B/L 1630 , and about 60 in NGC 2264 were found on deep, direct red plates taken by the SERC Schmidt telescope. The catalog of the HH candidates is being prepared with measuring machines at Kiso and supplementary observations to confirm the HH characteristics are on-going with using Anglo-Australian Telescope attached with a fiber spectrograph (0gura, private communication).

Acknowledgments. I thank Prof. T. Kogure and Dr. K. Ogura for sending me material in advance of publication.

\section{References}

Kogure, T., Yoshida, S., Wiramihardja, S. D., Nakano, M., Iwata, T. and Ogura, K. (1989) Pub. Astr. Soc. Japan 41, No. 6, in press.

Maddalena, R. B., Morris, M., Moscowitz, J. and Thaddeus, P. (1986) Astrophys. J. 303, 375.

Ogura, K. (1984) Pub. Astr. Soc. Japan 36, 139.

Ogura, K. (1987) IAU Symposium No. 115, Star Forming Regions, P. 341, ed. Peimbert, M. \& Jugaku, J., Reidel, Dordrecht, Holland.

Ogura, K. and Hasegawa, T. (1983) Pub. Astr. Soc. Japan 35, 299.

Ogura, K. and Hidayat, B. (1985) Pub. Astr. Soc. Japan 37, 537.

Ogura, K. and Walsh, J.R. (1989) Mon. Not. R. astr. Soc., to be submit ted.

Sato, F., Ogura, K. and Matsumoto, A. (1989) Pub. Astr. Soc. Japan, to be submitted.

Walsh, J.R. and Ogura, K. (1989) Mon. Not. R. astr. Soc., to be submit ted.

Warren, Jr., W. H. and Hesser, J.E. (1977) Astrophys. J. Suppl. 34, 115.

Wiramihardja, S. D., Kogure, T., Nakano. M. and Yoshida, S. (1986) Pub. Astr. Soc. Japan 38, 395.

Wiramihardja, S. D., Kogure, T., Yoshida, S., Ogura, K. and Nakano, M., (1989) Pub. Astr. Soc. Japan 41, 155. 
BLAAUW: Among the transparancies you showed was one of the Orion region exhibiting the molecular clouds and the various $O B$ subgroups, and there was also one showing equi-surface density curves of the emission line stars. I noted that there appears to be a correlation between the location of subgroups Ia and a feature in the emission line star density. This is interesting because this subgroup is almost void of interstellar matter and this implies that the subgroup Ia also contains low mass stars. 